\title{
FUCHSIAN GROUPS OF CONVERGENCE TYPE AND NONTANGENTIAL GROWTH OF AUTOMORPHIC FORMS
}

\author{
T. A. METZGER AND K. V. RAJESWARA RAO ${ }^{1}$
}

ABSTRACT. For a Fuchsian group, it is shown that the nontangential growth of automorphic forms characterizes whether or not the group is of convergence type. As a corollary of the main result, it is shown that for a Fuchsian group of second kind, not necessarily finitely generated, every integrable automorphic form of dimension -2 is zero, thus extending a known result.

1. Introduction and results. Let $\Gamma$ be a Fuchsian group acting on the unit disc $D$ of the complex plane $C$. Given any real number $q \geq 1$, we fix, once and for all, a set of factors of automorphy $\rho(q, T, z)(T \in \Gamma, z \in D)$ for $\Gamma$ (cf. [3]). Note that if $q$ is an integer, $\rho(q, T, z)=\chi(T) \cdot T^{\prime}(z)^{q}$, where $\chi$ is a character of $\Gamma$. A function $f$, holomorphic on $D$, is said to be an automorphic form of dimension $-2 q$ for $\Gamma$ if $f(T z) \rho(q, T, z)=f(z) \forall T \in$ $\Gamma, z \in D$.

Let $\lambda(z)|d z| \equiv|d z| /\left(1-|z|^{2}\right)$ be the Poincaré line element and $d \omega(z)$ $=\lambda^{2}(z) d x d y \quad(z=x+i y, x, y$ real $)$ be the corresponding area element on $D$. Let $\Omega$ be a fundamental region for $\Gamma$, in $D$, whose boundary has plane Lebesgue measure zero. An automorphic form $f$ of dimension $-2 q$ is said to be $p \cdot$ integrable if $f \cdot \lambda^{-q} \in L^{p}(\Omega, d \omega), 1 \leq p \leq \infty$, and the $L^{p}$-norm of $f \lambda^{-q}$ is denoted by $\|f\|_{p}$. With \|\|$_{p}$ as norm, the Banach space of all $p$ integrable automorphic forms is denoted by $A_{q}^{p}(\Gamma)$.

The group $\Gamma$ is said to be of convergence type if

$$
G(z)=\sum_{T \in \Gamma}\left(1-|T z|^{2}\right)<\infty
$$

for one, and hence all, $z$ in $D$. This is equivalent to the condition that $\Sigma_{T \in \Gamma}|\rho(1, T, z)|=\Sigma_{T \in \Gamma}\left|T^{\prime}(z)\right|<\infty$ for all $z$ in $D$, and also (cf. Tsuji $[7$, p. 522]) to the condition that the quotient Riemann surface $D / \Gamma$ be hyperbolic. The principal result of this note gives an estimate for the nontangential growth of $f \cdot \lambda^{-q}$ in terms of $G(z)$ for $f$ in $A_{q}^{p}(\Gamma)$ and is, essen-

Received by the editors September 18, 1973. 30 A98.

AMS (MOS) subject classifications (1970). Primary 30A58; Secondary 30 A52,

1 The authors were supported, in part, by the National Science Foundation. Copyright () 1975, American Mathematical Society 
tially, a consequence of an argument due to Gehring (cf. Tsuji [7, p. 187]).

Theorem 1. Let $\Gamma$ be of convergence type and $f \in A_{q}^{p}(\Gamma), 1 \leq p<\infty$. Then, for almost all $\theta$,

and, hence

$$
f(z) \lambda(z)^{-q}=o\left(G(z)^{1 / p}\right)
$$

as $z$ tends to $e^{i \theta}$ through any fixed Stolz angle with vertex at $e^{i \theta}$.

Remarks. If $\Gamma$ is not of convergence type and $f \not 0$, then (1.3) cannot be true for any set of $\theta$ of positive linear measure. For, then, let $z_{0} \in D$; then the orbit $\Gamma z_{0}$ clusters nontangentially at $e^{i \theta}$ for almost all $\theta$ (see [1, p. 52]), and if (1.3) were to hold for a set of $\theta$ of positive measure, then

$$
\left|f\left(z_{0}\right)\right| \cdot \lambda\left(z_{0}\right)^{-q}=\left|f\left(T z_{0}\right)\right| \cdot \lambda\left(T z_{0}\right)^{-q}=o(1),
$$

since $T z_{0}$ approaches $e^{i \theta}$ nontangentially almost everywhere. This implies that $f\left(z_{0}\right)=0$ for all $z_{0}$ in $D$, a contradiction. Thus, the Theorem provides a new characterization of Fuchsian groups of convergence type.

Corollary. Let $\Gamma$ be of second kind. Then $A_{1}^{1}(\Gamma)=\{0\}$.

Proof. Applying the Theorem with $p=q=1$, we conclude that, if $f \in$ $A_{1}^{1}(\Gamma)$, then for almost all $\theta, f(z)=o(G(z) \cdot \lambda(z))$ as $z \rightarrow e^{i \theta}$ through any fixed Stolz angle with vertex at $e^{i \theta}$. In view of Plessner's theorem (see Noshiro [5, p. 72]; cf, also, Tsuji [7, p. 339]), it thus suffices to show that, for a set of $\theta$ of positive (linear) Lebesgue measure, $G(z) \cdot \lambda(z)$ is bounded in any fixed Stolz angle with vertex at $e^{i \theta}$. However, $G(z) \cdot \lambda(z)=\Sigma_{T \in \Gamma}\left|T^{\prime}(z)\right|$ and this series converges uniformly on compact subsets of $C \backslash L$, where $L$ is the limit set of $\Gamma$. Thus $G(z) \cdot \lambda(z)$ is finite and continuous on $C \backslash L$. Recall that $L \subset$ the unit circle, and, if $\Gamma$ is of second kind, $C \backslash L$ contains an arc of the unit circle. The Corollary is now obvious.

Remarks. (1) The Corollary was known earlier (cf. [4] and, also, Knopp [2]) if $\Gamma$ is finitely generated and is of second kind. It also follows from rather standard harmonic majorization arguments, as pointed out to one of the authors orally by L. Carleson.

(2) Since for $\Gamma$ of convergence type, $G$ is bounded on $D[6$, pp. 636637], the present argument also shows that $A_{1}^{p}(\Gamma)=\{0\}$ if $p \leq 1$ and $\Gamma$ is of second kind.

2. Some lemmas. The proof of the Theorem depends on the following lemmas. 
Lemma l. Let $f \in A_{q}^{p}(\Gamma)$ and $\Gamma$ be of convergence type. Then,

$$
\|f\|_{p}^{p}=\iint_{D} \frac{\left|f(z) \lambda(z)^{-q}\right|^{p} d \omega(z)}{G(z) \cdot \lambda(z)}=\iint_{D} \frac{|f(z)|^{p}}{G(z)}\left(1-|z|^{2}\right)^{p q-1} d x d y .
$$

Proof. Indeed,

$$
\begin{aligned}
\iint_{D} \frac{\left|f(z) \lambda(z)^{-q}\right|^{p} d \omega(z)}{G(z) \lambda(z)} & =\sum_{i \in \Gamma} \int_{T} \int_{\Omega} \frac{\left|f(z) \lambda(z)^{-q}\right|^{p} d \omega(z)}{G(z) \cdot \lambda(z)} \\
& =\sum_{T \in \Gamma} \iint_{\mathbf{\Omega}} \frac{\left|f(z) \cdot \lambda(z)^{-q}\right|^{p} d \omega(z)}{G(T z) \lambda(T z)},
\end{aligned}
$$

since the numerator in the integrand is $\Gamma$-invariant. Since $G(T z) \cdot \lambda(T z) \cdot$ $\left|T^{\prime}(z)\right|=G(z) \cdot \lambda(z)=\Sigma_{S \in \Gamma}\left|S^{\prime}(z)\right|$, and the integrand is positive, the proof is completed by interchanging summation and integration.

Remark. If $\sigma_{\alpha}(z) \equiv \Sigma_{S \in \Gamma}\left|S^{\prime}(z)\right|^{\alpha}<\infty$, then, the same argument shows that

$$
\|f\|_{p}^{p}=\iint_{D} \frac{\left|f \lambda^{-q}\right|^{p} d \omega}{\sigma_{a}}
$$

Lemma 2. Let $f$ be analytic and $h$ be a positive, $C^{2}$ superharmonic function on $D$. If $t$ and $\alpha$ are positive numbers, then $|f|^{t} \cdot h^{-\alpha}$ is subharmonic on $D$.

Proof. Since $|f|^{t} \cdot h^{-a}$ is continuous on $D$ and the zeros of $f$ are isolated, it suffices to show that the Laplacian of $|f|^{t} b^{-a}$ is nonnegative whenever $f(z) \neq 0$, and then it suffices to consider the case $t=2$. Apart from a factor of 4 , this Laplacian

$$
\begin{aligned}
= & \frac{\partial^{2}}{\partial z \partial \bar{z}}\left(|f|^{2} h^{-a}\right)=\left|f^{\prime}\right|^{2} h^{-a}-2 R\left(\alpha \cdot f \cdot \bar{f}^{\prime} h^{-\alpha-1} \frac{\partial h}{\partial z}\right) \\
& +|f|^{2} a(\alpha+1) h^{-a-2}\left|\frac{\partial h}{\partial z}\right|^{2}-a|f|^{2} h^{-a-1} \frac{\partial^{2} h}{\partial z \partial \bar{z}} .
\end{aligned}
$$

Since, for $a$ and $b$ nonnegative, $a+b \geq 2 \sqrt{a b}$, it follows that, in the last expression, the sum of the first three terms $\geq 0$ and the last term is positive since $h$ is positive and superharmonic. This completes the proof.

Lemma 3. Let $w$ be a nonnegative subharmonic function on $D, m>1$ and $t>0$. If 


$$
\iint_{D} w^{m}(z) \cdot\left(1-|z|^{2}\right)^{t-1} d x d y<\infty
$$

then, for almost all $\theta, w(z)=o\left(\left(1-|z|^{2}\right)^{-t / m}\right)$ as $z \rightarrow e^{i \theta}$ through any fixed Stolz angle with vertex at $e^{i \theta}$.

Proof. As mentioned in the introduction, this proof is a simple modification of an argument due to Gehring (cf. Tsuji [7, p. 187]) who considered the case $t=1$. At the suggestion of the referee, we include the details.

For $0<\alpha<\pi / 2$, let $S^{a}(1, \theta)$ be the domain which is bounded by the two tangents from $e^{i \theta}$ to the circle $|z|=\sin \alpha$ and the larger arc of this circle which is bounded by the two points of contact. It suffices to show that, for almost all $\theta$,

$$
\lim _{z \rightarrow e^{i \theta}}\left(1-|z|^{2}\right)^{t} \cdot w^{m}(z)=0 \quad \text { as } z \rightarrow e^{i \theta} \text { in } S^{a}(1, \theta) \text {. }
$$

For $\sin a<r<1$, let $S_{r}^{a}(1, \theta)=\left\{z \in S^{a}(1, \theta)|| z \mid<r\right\}$ and $S^{\alpha}(R, \theta)$ $(0<R<1)$ be the image of $S^{a}(1, \theta)$ under the map $z \rightarrow R z$. We choose $R=$ $r^{1 / 2}$ and $\beta=\sin ^{-1} R$. Since $r<R<1$ and $R \sin \beta=r, S_{r}^{a}(1, \theta) \subset S^{\beta}(R, \theta)$. Thus, if $U(r, \theta)=\operatorname{Sup}_{z \in S_{r}^{\alpha}(1, \theta)} w(z), W(R, \theta)=\operatorname{Sup}_{z \in S^{\beta}(R, \theta)} w(z)$, then

$$
U(r, \theta) \leq W(R, \theta)
$$

and, to prove (2.2), it suffices to show that

$$
\lim _{r \rightarrow 1} U^{m}(r, \theta)\left(1-r^{2}\right)^{t}=0 .
$$

Since $m>1$, a result due to Hardy and Littlewood (cf. Tsuji [7, p. 185, Theorem IV, 39]) implies the existence of a finite constant $C_{1}(m, \alpha)$ such that

$$
\int_{0}^{2 \pi} W^{m}(R, \theta) d \theta \leq C_{1}(m, \alpha) \int_{0}^{2 \pi} w^{m}\left(R e^{i \theta}\right) d \theta .
$$

This and (2.3) imply that

$$
I \equiv \int_{\sin \alpha}^{1} \int_{0}^{2 \pi} U^{m}(r, \theta)\left(1-r^{2}\right)^{t-1} r d r d \theta
$$

$$
\leq C_{1}(m, \alpha) \int_{\sin \alpha}^{1} \int_{0}^{2 \pi} w^{m}\left(R e^{i \theta}\right)\left(1-r^{2}\right)^{t-1} r d r d \theta .
$$

Recalling that $R=r^{1 / 2}$ and making the obvious change of variable, we conclude the existence of a finite constant $C_{2}(m, \alpha, t)$ such that

$$
I \leq C_{2}(m, a, t) \iint_{D} w^{m}(z)\left(1-|z|^{2}\right)^{t-1} d x d y .
$$


By (2.1), I is then finite so that for almost all $\theta, \int_{0}^{1} U^{m}(r, \theta)\left(1-r^{2}\right)^{t-1} r d r<$ $\infty$. Thus, for almost all $\theta$,

$$
\lim _{r \rightarrow 1} \int_{r}^{1} U^{m}(s, \theta)\left(1-s^{2}\right)^{t-1} s d s=0 .
$$

Since $U(r, \theta)$ increases with $r$, this implies (2.4), thus completing the proof.

3. Proof of the Theorem. Let $f \in A_{q}^{p}(\Gamma)$. Since $G$, evidently, is positive, $C^{2}$ and superharmonic, Lemma 2 implies that $|f|^{s} G^{-\beta}$ is subharmonic in $D$, for all positive constants $s$ and $\beta$. Noting Lemma 1 , we then apply Lemma 3 with $t=p q$ and $m=p, w=|f| G^{-1 / p}$ if $p>1$ and $m=1 / \alpha, w=|f|^{\alpha} G^{-\alpha}$, $0<a<1$, if $p=1$. In either case, Lemma 3 then implies that, for almost all $\theta, f(z) G(z)^{-1 / p}=o\left(\left(1-|z|^{2}\right)^{-q}\right)$ as $z \rightarrow e^{i \theta}$ through any Stolz angle with vertex at $e^{i \theta}$. This is equivalent to (1.2), thus completing the proof of one half of the Theorem.

To prove the other half, it suffices to show that the nontangential limit of $G(z)$ is zero at $e^{i \theta}$ for almost all $\theta$. Since $1-x \leq-\log x(|x|<1)$, it follows that

$$
0 \leq G(z) \leq-2 \sum_{T \in \Gamma} \log |T z|=-2 \log |B(z)|,
$$

where $B(z)$ is "the" Blaschke product with zeros at $T^{-1} 0, T \in \Gamma$. Since $\Gamma$ is of convergence type, clearly $B(z)$ exists and $\log |B(z)|$ has nontangential limit zero at $e^{i \theta}$ for almost all $\theta$. In view of (3.1), this completes the proof of the Theorem.

\section{REFERENCES}

1. C. Constantinescu, Über die Klassifikation der Riemannschen Flächen, Acta Math. 102 ( 1959), 47-78. MR 22 \#12219.

2. M. I. Knopp, Bounded and integrable automorphic forms, Indiana Univ. Math. J. 22 (1973), 769-778.

3. T. A. Metzger and K. V. Rajeswara Rao, On integrable and bounded automorphic forms, Proc. Amer. Math. Soc. 28 (1971), 562-566. MR 43 \#6432.

4. - On integrable and bounded automorphic forms. II, Proc. Amer. Math. Soc. 32 (1972), 201-204. MR 45 \#2164.

5. K. Noshiro, Cluster sets, Ergebnisse der Mathematik und ihrer Grenzgebiete, N.F., Heft 28, Springer-Verlag, Berlin, 1960. MR 24 \#A3295.

6. K. V. Rajeswara Rao, Fuchsian groups of convergence type and Poincaré series of dimension -2, J. Math. Mech. 18 (1968/69), 629-644. MR 38 \#6065.

7. M. Tsuji, Potential theory in modern function theory, Maruzen, Tokyo, 1959. MR $22 \# 5712$.

DEPARTMENT OF MATHEMATICS, UNIVERSITY OF PITTSBURGH, PITTSBURGH, PENNSYLVANIA 15213

DIVISION OF MATHEMATICAL SCIENCES, PURDUE UNIVERSITY, WEST LAFAYETTE, INDIANA 47907 